\title{
A COMPARISON OF BRACKET FAILURE RATE BETWEEN DIRECT AND INDIRECT BONDING TECHNIQUES: A PROSPECTIVE CLINICAL STUDY
}

\author{
Ahmed Basheer Ayyed ${ }^{*}$, Ramadan Yousef Abu Shahba**, Mohamed Helmi Saleh**, Farouk Hussain**
}

\begin{abstract}
Objective: The aim of the present study was to make a clinical comparison of bracket position accuracy and failure rate between direct and indirect bonding techniques. Subjects and methods: Thirty patients were selected. Patients divided into two groups according to the technique, each group consist of 15 patients. Group A included the patients that treated with direct bonding technique. Group B included the patients that treated with indirect bonding technique. Check-up was carried out every 3 weeks and a special sheet was used to record the failure rate of the brackets. Results: 294 brackets were bonded in a group A (direct technique), and only 39 brackets were debonded. 290 brackets were bonded in a group B (indirect technique), and only 47 brackets were debonded. Conclusion: Generally, there is not clinically significance in the failure rate between direct and indirect bonding techniques.
\end{abstract}

KEYWORDS: Bracket, failure, direct, indirect, bonding.

\section{INTRODUCTION}

One of the aims of orthodontics is improving the dental health; this is achieved through positioning the teeth into the best functional balance. Another aim is to obtain the maximum esthetical outcome for the patient. By far the most commonly used appliance in fixed orthodontic treatment is the dental brackets ${ }^{(1)}$.

There are numerous techniques regarding the bonding of the brackets, including direct and indirect bonding techniques. Traditional direct brackets placement involves placing each bracket directly on the tooth by operator. While the indirect bonding technique is executed by attaching the brackets on dental casts in the laboratory then transferring these brackets to the patient's teeth through transferring trays ${ }^{(2)}$.
Indirect bonding method became very popular after it was first described by Silverman and Cohen in 1972. This technique was proposed due to its many advantages, it is more time-saving as it optimizes the use of the orthodontist's time, removal of flash to the bracket bases which can promote plaque and calculus formation. In addition to minimizing the patient's discomfort and increasing the stability of the treatment as the most valuable goal of orthodontic treatment is to create permanent results. The indirect bonding technique play an important role in enabling the clinician to modify the bracket position according to the patient's need especially in deep-bite cases ${ }^{(2-12)}$.

There are various techniques of indirect bonding, according to bracket base preparation (standard or customized), transfer mask type (single jigs or full arch) and transfer tray material (acrylic resin, silicone, thermo-printed material) ${ }^{(2,14,16)}$.

\footnotetext{
* Graduate Students, Department of Orthodontics, Faculty of Dentistry, Al Azhar University

** Assistant Professor, Department of Orthodontics, Faculty of Dentistry, Al Azhar University
} 
Brackets failure considered to be a major concern during orthodontic treatment with fixed appliances, as it can be upsetting and, in some instances, critical in the overall success of the treatment. The detachment of the brackets during corrective procedures may also lead to an increase in the duration of the treatment, damage to tooth enamel, and increased chairside-time due to re-bonding procedure. Hence, it could also elevate the costs of the overall orthodontic treatment, consequently multiple studies have reported varying incidence of bracket failure following orthodontic brackets bonding. Multiple studies have reported varying incidence of bracket failure following orthodontic brackets bonding, while other studies compared various techniques of orthodontic bonding and rates of brackets failure. ${ }^{(16-26)}$

The indirect technique was considered inferior due to the high number of brackets lost, in addition to the increased time that required for procedure of bracket placement and removal of excess adhesive flash around the bracket bases. The reasons of that high failure rate were thought to be due to the chemically-cured composite adhesive that was used and it may back to the variations of the technique. Several studies have been conducted in order to evaluate the effectiveness of indirect technique. Hence, only few reports evaluated the clinical reliability of the indirect bonding technique compared with the direct bonding technique ${ }^{(2,24)}$.

This study aimed to create a make a clinical comparison of failure rate between direct and indirect bonding techniques.

\section{SUBJECTS AND METHODS}

The design of this study was a randomized clinical study. The unit of analysis and randomization is the individual patient. This longitudinal study was conducted on patients seeking orthodontic treatment in the outpatient clinic, Faculties of Dental Medicine, Al-Azhar University, Cairo, Boys and Girls Branches. Thirty orthodontic patients both males and females are selected and treated by the researcher. Sample size calculation was undertaken according to previous study.

Inclusion criteria: The age should be ranging between 14 and 24 years, also both sexes are included. This orthodontic treatment should be the first orthodontic treatment for the patient. The patients should have a good general health and oral hygiene. The patients should have a permanent dentition with minimal to moderate crowding. Also, the patients should have normal enamel.

Exclusion criteria: Patients that may need an extra oral appliance, also the patients have retained deciduous teeth or having dental morphogenesis anomalies. Patients with attrition, fractured/restored incisal edges or cusp-tips. Uncooperative patients who miss two successive appointments or didn't follow the operator's instructions. The patients have systemic or genetic disease that could interfere with orthodontic treatment.

Ethical considerations: An informed consent form that explains every step in the research was given and discussed carefully with the patients before participation in the study and was signed freely. The objectives of the study were discussed and explained with the patients and / or guardians as well.

Patient allocation: The patients were randomly divided equally into two groups according to the type of bonding technique that was used. Group A (direct bonding group) this group included 15 patients treated with direct bonding technique. Group B (Indirect bonding group) this group include 15 patients treated with indirect bonding technique.

Preparation for bonding: According to previous study, a similar method was employed to prepare the teeth for bonding whether a direct or indirect technique was to be used. Each arch of teeth 
was prepared and bonded separately to decrease the risk of moisture contamination. Teeth were polished for five seconds each using a bristle brush in a slow speed hand piece with a slurry of pumice and water. The teeth were then rinsed with an air/water spray until all traces of pumice had been totally removed. A cheek retractor and a flexible saliva ejector were used to provide a control for moisture, and cotton wool rolls were placed in the buccal and lingual sulci to improve isolation. The teeth were then dried with oil-free compressed air for $5 \mathrm{~s}$ each and etched for 20 s a with $37 \%$ phosphoric acid*, in accordance with the manufacturer's instructions. Each tooth was rinsed thoroughly for $15 \mathrm{~s}$ until all the traces of the blue etching gel were removed before they were dried again with oil-free compressed air until they exhibited a frosty white appearance with no traces of moisture.

The indirect bonding technique (Impression taking stage): A plastic tray was used for this procedure, first the operator got ensure that the tray is totally fitting patient arch without any interference. Condensation silicones** was used as an impression material to ensure that there were not dimensionally changes may take place.

\section{The indirect bonding technique (laboratory} stage): Models were cast on the same day as impression taking to ensure accurate fit of the transfer trays and trimmed to allow easy use of the vacuum forming machine. The appropriate bracket (Versatile Roth American orthodontics - Low profile master series.) was selected for each tooth and a small amount of composite material (3M Composite TransXT), was placed onto the base. The separating medium (Sphinx Alexandria) applied on the teeth and left for 7 minutes to get dried. Each bracket was then positioned on its tooth and the adhesive was light cured after fully insurance that the bracket placed in it's ideal position, using light cure device (Woodpecker Curing light led.B). Trays were made using a $.040 "(1 \mathrm{~mm})$ thick blank of transparent tray material. A square blank was draped over a dry model and brackets. The blank was first heated and then closely adapted to the model by means of negative pressure using a vacuum forming apparatus. After cooling of the model, it trimmed with a hot instrument and removed from the model along with the brackets that were contained within it. The bases of the bracket got cleaned using distilled water and tooth-brush. Finally, the tray was trimmed to be ready for clinical stage (fig. 1).

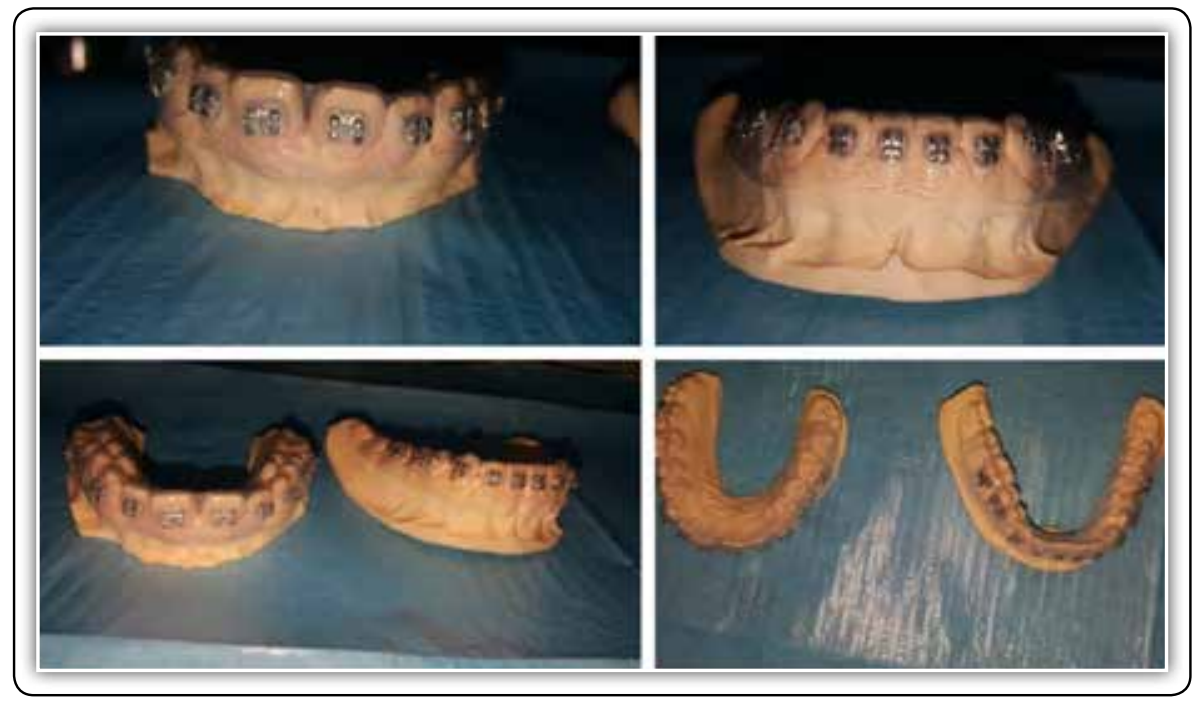

FIG (1) Casts after trimming the trays. 
The indirect bonding technique (clinical stage): The teeth were prepared as mentioned before. A thin layer of adhesive (3M Transt XT bond unitek) was applied to the bracket bases and to the teeth in the quadrant to be indirectly bonded. A small amount of light cure orthodontic adhesive was placed onto the base of each bracket and the tray was seated with even pressure to allow good adaptation of the brackets to the teeth and an even thickness of composite resin. Care was taken to place a minimum amount of composite resin onto each bracket base to avoid excessive adhesive flash. Each bracket was cured using same light curing device that it mentioned before for $20 \mathrm{~s}, 10 \mathrm{~s}$ on the mesial and $10 \mathrm{~s}$ on the distal aspect. Brackets (Versatile Roth American orthodontics - Low profile master series) were cured starting with the most posterior tooth, then moving forwards and the tray was then carefully removed using a flat plastic instrument. Excessive adhesive flash was removed using rotary instruments if necessary.

Direct bonding technique: The teeth were prepared as it mentioned before and adhesive bond (3M Transt XT bond) was then painted onto each tooth and bracket base. A small amount of adhesive composite3) M Composite TransXT) was applied to each bracket base and the bracket was then positioned onto the LA point of the tooth. All brackets in the quadrant were positioned and excess composite was removed before the curing light was applied. Each bracket was cured for $20 \mathrm{~s}, 10 \mathrm{~s}$ on the mesial and $10 \mathrm{~s}$ on the distal aspect. To minimize variation in the magnitude of orthodontic forces applied to the teeth, a similar initial 0.012-inch nickel titanium (Ortho Organizers Inc.) arch wire was used in each case, $16 \times 22$ wire will be the last wire to use in the end of levelling and alignment stage. At each visit, a record was kept of the tooth type, date and circumstances of bracket bond failures. All subjects were observed over a period of levelling and alignment.
Observations: All patients were provided with oral instruction written on a sheet for maintenance of their fixed appliance. In addition, the operator explained the instruction in detail to the patient. Check-up was carried out every 3 weeks. The patients were recommended to inform the dentist immediately if suspecting a detachment, as all of that information will be written in special sheet. Detachment date was registered, and the bracket was replaced with a new one; in group B, a section of the transfer tray used for the initial bonding has been used for repositioning when necessary.

\section{RESULTS}

The term of anterior segment indicates the total of central, lateral incisors and canine, while the term of posterior segment indicates the total of 1 st and 2 nd premolars. Data were collected, revised, coded and entered to the Statistical Package for Social Science (IBM SPSS) version 23. The distribution of quantitative data was tested by Kolmogorov-Smirnov test of normality. So, the quantitative data were presented as mean, standard deviations and ranges. Also, qualitative variables were presented as number and percentages as it shows in tables 1 and 2 .

The comparison between groups regarding qualitative data was done by using Chi-square test and/or Fisher exact test when the expected count in any cell found less than 5. The comparison between two independent groups with quantitative data and parametric distribution was done by using Independent t-test while the comparison between two independent groups regarding quantitative data with non-parametric distribution were done by using Mann-Whitney test. The confidence interval was set to $95 \%$ and the margin of error accepted was set to $5 \%$. So, the p-value was considered significant as the following:

$$
\begin{aligned}
& \text { P-value }>0.05: \text { Non significant }(\mathrm{NS}) \\
& \text { P-value }<0.05: \text { Significant }(\mathrm{S}) \\
& \text { P-value }<0.01 \text { : Highly significant }(\mathrm{HS})
\end{aligned}
$$


TABLE (1) Comparison of failure rate of right central incisors, lateral incisors, canines, $1^{\text {st }}$ premolars, $2^{\text {nd }}$ premolars, anterior and premolar segments in both upper and lower arches between group A and group B.

\begin{tabular}{|l|c|c|c|c|c|c|c|}
\hline \multirow{2}{*}{ Right } & \multicolumn{2}{|c|}{ Group A } & \multicolumn{2}{c|}{ Group B } & \multirow{2}{*}{ Test value } & P-value & Sig. \\
\cline { 2 - 7 } & No. & $\%$ & No. & $\%$ & & & \\
\hline Upper central incisor & 2 & $13.3 \%$ & 0 & $0.0 \%$ & 2.143 & 0.143 & NS \\
\hline Upper lateral incisor & 0 & $0.0 \%$ & 0 & $0.0 \%$ & 0.000 & 1.000 & NS \\
\hline Upper canine & 0 & $0.0 \%$ & 0 & $0.0 \%$ & 0.000 & 1.000 & NS \\
\hline Upper anterior segment & 2 & $13.3 \%$ & 0 & $0.0 \%$ & 2.143 & 0.143 & NS \\
\hline Upper 1 $1^{\text {st }}$ premolar & 2 & $13.3 \%$ & 5 & $33.3 \%$ & 1.677 & 0.195 & NS \\
\hline Upper 2 ${ }^{\text {nd }}$ premolar & 4 & $26.7 \%$ & 4 & $26.7 \%$ & 0.000 & 1.000 & NS \\
\hline Upper posterior segment & 6 & $40.0 \%$ & 9 & $60.0 \%$ & 1.200 & 0.273 & NS \\
\hline Lower central incisor & 0 & $0.0 \%$ & 1 & $6.7 \%$ & 1.034 & 0.309 & NS \\
\hline Lower lateral incisor & 0 & $0.0 \%$ & 0 & $0.0 \%$ & 0.000 & 1.000 & NS \\
\hline Lower canine & 0 & $0.0 \%$ & 2 & $13.3 \%$ & 2.143 & 0.143 & NS \\
\hline Lower Anterior segments & 0 & $0.0 \%$ & 3 & $20.0 \%$ & 3.333 & 0.067 & NS \\
\hline Lower 1 $1^{\text {st }}$ premolar & 4 & $26.7 \%$ & 6 & $40.0 \%$ & 0.600 & 0.438 & NS \\
\hline Lower 2 $2^{\text {nd }}$ premolar & 6 & $40.0 \%$ & 9 & $60.0 \%$ & 1.200 & 0.273 & NS \\
\hline Lower posterior segments & 10 & $66.7 \%$ & 15 & $100.0 \%$ & 6.000 & 0.014 & S \\
\hline
\end{tabular}

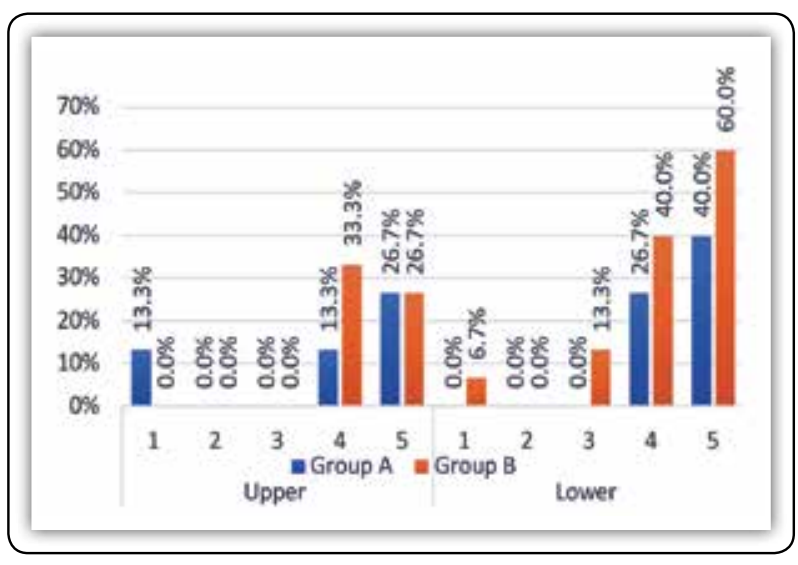

FIG (2) Comparison of failure rate of right central incisors, lateral incisors, canines, and 1 st and 2nd premolars in both upper and lower arches between group A and group B.

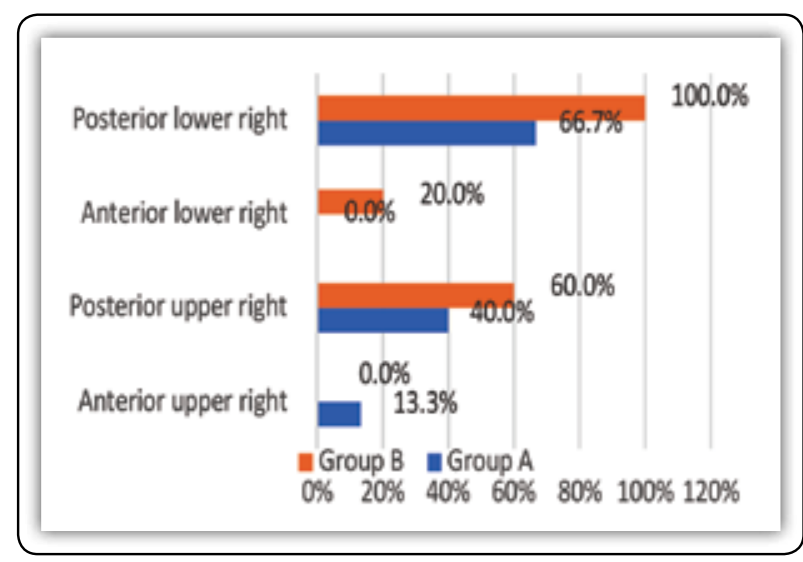

FIG (3) Comparison of failure rate of right anterior and premolar segments in both upper and lower arches between group A and group B. 
TABLE (2) Comparison of failure rate of left central incisors, lateral incisors, canines, 1st premolars, 2nd premolars, anterior and posterior segments in both upper and lower arches between group A and group B.

\begin{tabular}{|c|c|c|c|c|c|c|c|}
\hline \multirow{2}{*}{ Left } & \multicolumn{2}{|c|}{ Group A } & \multicolumn{2}{|c|}{ Group B } & \multirow{2}{*}{ Test value } & \multirow{2}{*}{ P-value } & \multirow{2}{*}{ Sig. } \\
\hline & No. & $\%$ & No. & $\%$ & & & \\
\hline Upper central incisor & 3 & $20.0 \%$ & 0 & $0.0 \%$ & 3.333 & 0.067 & NS \\
\hline Upper lateral incisor & 2 & $13.3 \%$ & 0 & $0.0 \%$ & 2.143 & 0.143 & NS \\
\hline Upper canine & 1 & $6.7 \%$ & 0 & $0.0 \%$ & 1.034 & 0.309 & NS \\
\hline Anterior upper segment & 6 & $40.0 \%$ & 0 & $0.0 \%$ & 7.500 & 0.006 & HS \\
\hline Upper $1^{\text {st }}$ premolar & 2 & $13.3 \%$ & 3 & $20.0 \%$ & 0.240 & 0.624 & NS \\
\hline Upper $12^{\text {nd }}$ premolar & 4 & $26.7 \%$ & 3 & $20.0 \%$ & 0.186 & 0.666 & NS \\
\hline Posterior upper segment & 6 & $40.0 \%$ & 6 & $40.0 \%$ & 0.000 & 1.000 & NS \\
\hline Lower central incisor & 0 & $0.0 \%$ & 0 & $0.0 \%$ & 0.000 & 1.000 & NS \\
\hline Lower lateral incisor & 2 & $13.3 \%$ & 1 & $6.7 \%$ & 0.370 & 0.543 & NS \\
\hline Lower canine & 0 & $0.0 \%$ & 0 & $0.0 \%$ & 0.000 & 1.000 & NS \\
\hline Anterior lower segment & 2 & $13.3 \%$ & 1 & $6.7 \%$ & 0.370 & 0.543 & NS \\
\hline Lower $1^{\text {st }}$ premolar & 0 & $0.0 \%$ & 7 & $46.7 \%$ & 9.130 & 0.002 & HS \\
\hline Lower $2^{\text {nd }}$ premolar & 7 & $46.7 \%$ & 6 & $40.0 \%$ & 0.136 & 0.712 & NS \\
\hline Posterior lower segment & 7 & $46.7 \%$ & 13 & $86.7 \%$ & 5.400 & 0.020 & $\mathrm{~S}$ \\
\hline
\end{tabular}

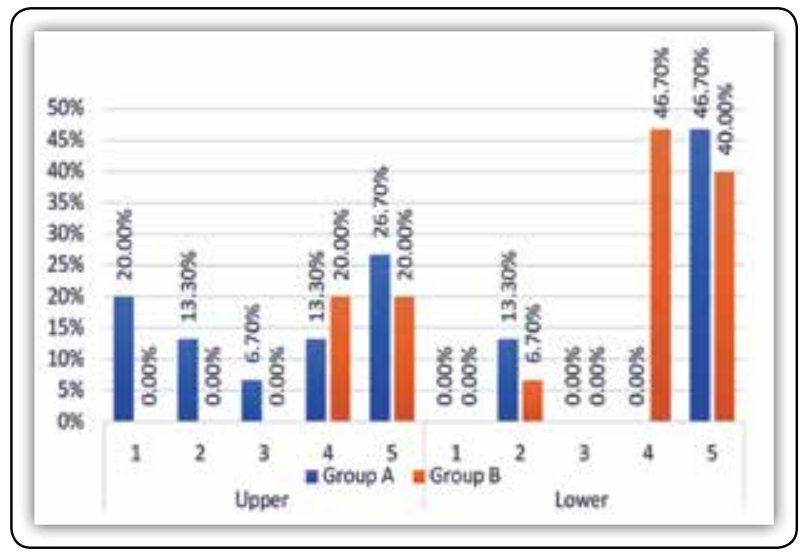

FIG (4) Comparison of failure rate of left central incisors, lateral incisors, canines, 1 st premolars and 2 nd premolars in both upper and lower arches between group A and group B.

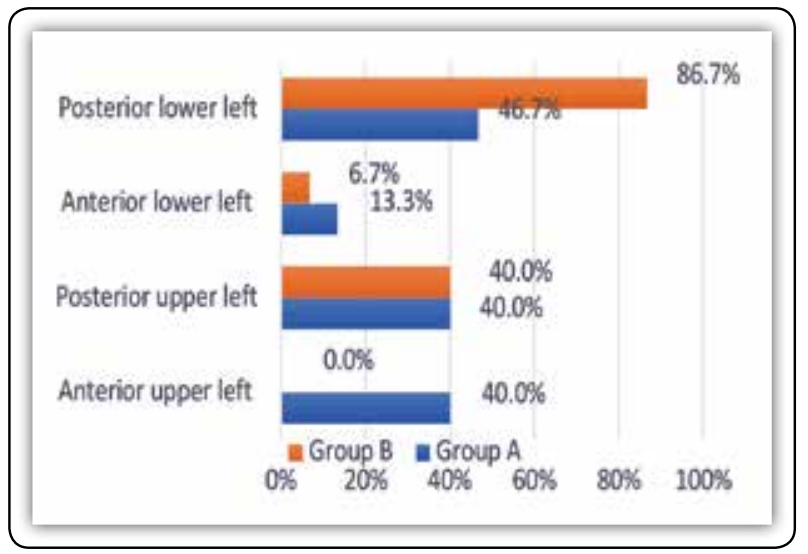

FIG (5) Comparison of failure rate of left anterior and posterior segments in both upper and lower arches between group A and group B. 


\section{DISCUSSION}

The aim of this study was also to measure, evaluate, and compare the failure rate between direct and indirect bonding techniques. The present study found that when using the indirect technique, the bonding failure rate is not significantly different when the direct technique is used, these results are in agreement with Deahl et al., Anna et al., and Thiyagarajah et al ${ }^{(27-29)}$.

Respectively, the results of this study differ from the finding of Zachrisson and Brobakken who reported a failure rate of $14 \%$ for indirect bonding and $2.5 \%$ for the direct method, the difference (2.5 percent versus 13.9 percent) was statistically significant $(\mathrm{P}<0.01)$. However, it is difficult to make direct comparisons since this last study used four different combinations of bonding techniques, adhesives and bracket bases for each patient ${ }^{(30)}$.

In the present study the age of the patient and the level of crowding was determined in the inclusion criteria, however the present study didn't put a consideration for the gender of the patient. Anna et al. in their study they didn't evaluate gender, age and type of malocclusion. Conflicting reports are present in the literature concerning these variables: some authors found significant differences in bond failure rate in patients with different malocclusions, age and gender. Other found the same results in male and female, patients with different age and malocclusion ${ }^{(29,31,32)}$.

The present study also shows the posterior lower left segments showed significant difference between the two groups. This may be attributed to the ideal position of brackets may lead to interference with opposing teeth, or it may be attributed to the short clinical crowns of the lower premolars and consequently the position of the brackets of these teeth subjected to pressure exerted by the opposing upper teeth, which may be due to differences of nature of malocclusion.
Linklater et al. reported that the loose brackets may have been caused by failure during laboratory step of group B, poor tooth polishing, failure during acid etching, poor control of humidity, several contaminations (saliva and blood), improper occlusion (contact of the opposing with orthodontic brackets), repeated trauma (e.g. hard food) and even lastly enamel quality ${ }^{(33)}$.

The results of this study suggest that the bonding failure rate is comparable in practice that routinely use these bonding techniques. Therefore, there should be no long-term difference in the bonding failure rate when considering the advantages and disadvantages of indirect bonding. Due to the presence of multiple variables that affect both bonding failure rate and treatment efficiency, it is critical to evaluate these across several studies. However, if we put in our consideration the sample size, it will show more reliability.

\section{CONCLUSION}

Generally, there is not clinically significance in the failure rate between direct and indirect bonding techniques.

\section{REFERENCES}

1. Murakami T1, Kawanabe N, Kataoka T, Hoshijima M, Komori H, Fujisawa A, Kamioka H.A Single-center, Openlabel, Randomized Controlled Clinical Trial to Evaluate the Efficacy and Safety of the Indirect Bonding Technique. Acta Med Okayama. 2016;70:413-6.

2. Menini A, Cozzani M, Sfondrini MF, Scribante A, Cozzani P, Gandini P. A 15-month evaluation of bond failures of orthodontic brackets bonded with direct versus indirect bonding technique: a clinical trial. Prog Orthod. 2014; $15: 70$

3. Kalange JT. Indirect bonding: a comprehensive review of the advantages. World J Orthod. 2004;4:301-7.

4. White LW. A new and improved indirect bonding technique. J Clin Orthod. 1999;33:17-23.

5. Fortini A, Giuntoli F, Franchi L. A simplified indirect bonding technique. J Clin Orthod. 2007;11:680-3. 
6. Hodge TM, Dhopatkar AA, Rock WP, Spary DJ. The Burton approach to indirect bonding. J Orthod. 2001;28:267-70.

7. Cozzani M, Menini A, Bertelli A. Etching masks for precise indirect bonding. J Clin Orthod. 44:326-30.

8. Kalange JT, Thomas RG. Indirect bonding: a comprehensive review of the literature. Semin Orthod. 2007;13:3-10.

9. Sinha PK, Nanda RS, Ghosh J. A thermal-cured, fluoride-releasing indirect bonding system. J Clin Orthod. 1995;29:97-100.

10. Pamukçu H, Özsoy ÖP. Indirect Bonding Revisited. Turk J Orthod. 2016;29(3):80-6.

11. Boese LR. Fiberotomy and reproximation without lower retention, nine years in retrospec: Part I. Angle Orthod. 1980;50:88-97.

12. Kalange JT. İndirect Bonding: A comprehensive review of the advantages. World J Orthod. 2004;5:301-7.

13. Aguirre MJ, King GJ, Waldrom MJ. Assessment of bracket placement and bond strength when comparing direct bonding technique. Am J Orthod. 1982;82:269-76.

14. Dalessandri D, Dalessandri M, Bonetti S, Visconti L, Paganelli C. Effectiveness of an indirect bonding technique in reducing plaque accumulation around braces. Angle Orthod. 2012;82:313-8.

15. Zachrisson B, Brobakkeen B. Clinical comparison of direct versus indirect bonding, with different bracket types adhesives. Am J Orthod. 1978;74:62-78.

16. Almosa N, Zafar H. Incidence of orthodontic brackets detachment during orthodontic treatment: A systematic review. Pak J Med Sci. 2018;34(3):744-50.

17. Bishara SE, VonWald LBA, Laffoon JF, Warren JJ. The effect of repeated bonding on the shear bond strength of a composite resin orthodontic adhesive. Angle Orthod. 2000;70(6):435-41.

18. Attishia R, Van Sickels JE, Cunningham LL. Incidence of bracket failure during orthognathic surgery:a comparison of two techniques to establish interim maxillomandibular fixation. Oral Maxillofac Surg. 2015;19(2):143-7.

19. Talic NF. Effect of fluoridated paste on the failure rate of precoated brackets bonded with self-etching primer:a prospective split-mouth study. Am J Orthod Dentofacial Orthop. 2011;140(4):527-30.

20. Krishnaswamy NR, Sunitha C. Light-emitting diode vs halogen light curing of orthodontic brackets:a 15-month clinical study of bond failures. Am J Orthod Dentofacial Orthop. 2007;132(4):518-23.
21. Varlik SK, Demirbas E. Effect of light-cured filled sealant on the bond failure rate of orthodontic brackets in vivo. Am J Orthod Dentofacial Orthop. 2009;135(2):144.e1-4. Discussion 144-145.

22. Bovali E, Kiliaridis S, Cornelis MA. Indirect vs direct bonding of mandibular fixed retainers in orthodontic patients:a single-center randomized controlled trial comparing placement time and failure over a 6-month period. Am J Orthod Dentofacial Orthop. 2014;146(6):701-8.

23. Cacciafesta V, Sfondrini MF, Melsen B, Scribante A. A 12 month clinical study of bond failures of recycled versus new stainless steel orthodontic brackets. Eur J Orthod. 2004;26(4):449-54.

24. Thiyagarajah S, Spary DJ, Rock WP. A clinical comparison of bracket bond failures in association with direct and indirect bonding. J Orthod. 2006;33(3):198-204.

25. Chapman JL. Bond failure rates of two self-ligating brackets:a randomised clinical trial. Aust Orthod J. 2011;27(2):139-44.

26. Bazargani F, Magnuson A, Lothgren H, Kowalczyk A. Orthodontic bonding with and without primer:a randomized controlled trial. Eur J Orthod. 2016;38(5):503-507.

27. Deahl ST, Salome N, Hatch JP, Rugh JD. Practice-based comparison of direct and indirect bonding. Am J Orthod Dentofacial Orthop. 2007;132:738-42.

28. Thiyagarajah S, Spary DJ, Rock WP. A clinical comparison of bracket bond failures in association with direct and indirect bonding. J Orthod. 2006;33:198-204.

29. Menini A, Cozzani M, Sfondrini MF, Scribante A, Cozzani P, Gandini P. A 15-month evaluation of bond failures of orthodontic brackets bonded with direct versus indirect bonding technique: a clinical trial. Prog Orthod. 2014;15:70.

30. Zachrisson BU, Brobakken BO. Clinical comparison of direct versus indirect bonding with different bracket types and adhesives. Am J Orthod. 1978;74:62-78.

31. Millett DT, McCluskey LA, McAuley F, Creanor SL, Newell J, Love J. A comparative clinical trial of a compomer and a resin adhesive for orthodontic bonding. Angle Orthod. 2000;70:233-340.

32. Millett DT, Gordon PH. A 5-year clinical review of bond failure with a no-mix adhesive (Right on?). Europ J Orthod. 1994;16:203-211.

33. Linklater RA, Gordon PH. Bond failure patterns in vivo. Am J Orthod Dentofacial Orthop. 2003;123(4):534-9. 